\title{
Therapeutic strategy for non-small-cell lung cancer patients with brain metastases (Review)
}

\author{
YOUNG HAK KIM, HIROKI NAGAI, HIROAKI OZASA, YUICHI SAKAMORI and MICHIAKI MISHIMA \\ Department of Respiratory Medicine, Graduate School of Medicine, Kyoto University, Kyoto 606-8507, Japan
}

Received May 28, 2013; Accepted July 08, 2013

DOI: 10.3892/br.2013.151

\begin{abstract}
Brain metastases are frequently encountered in patients with non-small-cell lung cancer (NSCLC) and are a significant cause of morbidity and mortality. Chemotherapy has been deemed ineffective under the hypothesis that the blood-brain barrier (BBB) limits the delivery of chemotherapeutic agents to the brain. Thus, radiotherapy and occasionally surgery have been selected for the treatment of brain metastases. However, recent clinical data suggested that chemotherapy may be an effective treatment option for patients with brain metastases, since patients who have developed brain metastases may have an inherently compromised BBB. The prognosis of NSCLC patients with brain metastases is generally poor and more effective treatment is required to improve their prognosis. Bevacizumab (Avastin) is a humanized monoclonal antibody that inhibits tumor angiogenesis by neutralizing the vascular endothelial growth factor. Preclinical data indicated that bevacizumab may be effective in preventing as well as treating preexisting brain metastases. Although safety concerns regarding intracranial hemorrhage have been a barrier for the use of bevacizumab in patients with brain metastases, safety data have gradually been accumulated through recent clinical trials. In this review, we aimed to summarize the currently available treatment options and present a therapeutic strategy for NSCLC patients with brain metastases, with a special emphasis on bevacizumab.
\end{abstract}

\section{Contents}

1. Introduction

2. Whole-brain radiotherapy

3. Surgery

4. Radiosurgery

Correspondence to: Dr Young Hak Kim, Department of Respiratory Medicine, Graduate School of Medicine, Kyoto University, 54 Shogoin-Kawaharacho, Sakyo-ku, Kyoto 606-8507, Japan E-mail: ekim@kuhp.kyoto-u.ac.jp

Key words: non-small-cell lung cancer, brain metastases, surgery, whole-brain radiotherapy, stereotactic radiotherapy, chemotherapy, bevacizumab

\author{
5. Chemotherapy \\ 6. Bevacizumab \\ 7. Treatment algorithm for NSCLC patients with brain \\ metastases \\ 8. Conclusions
}

\section{Introduction}

Non-small-cell lung cancer (NSCLC) is the leading cause of cancer-related mortality in several industrialized countries and its incidence is increasing worldwide. It is estimated that $40 \%$ of patients with newly diagnosed NSCLC have incurable stage IV disease (1). The remaining patients receive radical treatment, such as surgery or chemoradiotherapy. However, approximately half of the patients who receive surgery eventually relapse and, among the patients who receive chemoradiotherapy, only a small proportion are cured. Consequently, the majority of NSCLC patients are eventually classified as having stage IV disease.

Although exact data are unavailable, the incidence of brain metastases in NSCLC patients is reportedly $24-44 \%$ and it is considered to be increasing with the advances in diagnostic techniques, such as magnetic resonance imaging (2). In a retrospective review of 809 patients with NSCLC and brain metastases, 181 patients $(22 \%)$ had brain metastases at initial staging, of whom 61 (34\%) were asymptomatic. Patients with non-squamous NSCLC had a significantly higher risk of brain metastases compared to patients with squamous cell NSCLC (3).

The available survival data on NSCLC patients with brain metastases are limited, since such patients are generally excluded from clinical trials. However, previous retrospective analyses reported that the median survival time (MST) of such patients is 3-6 months (4-6). According to a recent retrospective analysis (7) of 81 patients with brain metastases who were diagnosed between January, 1996 and June, 2007, the MST was 5 months and the 1- and 2-year survival rates were 14 and $7.6 \%$, respectively. In addition, neurologically symptomatic patients exhibited significantly shorter survival compared to asymptomatic patients, with an MST of 4.0 and 7.5 months, respectively $(\mathrm{P}=0.02)(7)$.

At present, there are three available treatment options for NSCLC patients with brain metastases: surgery, radiotherapy and chemotherapy. These therapeutic approaches should be selected appropriately, based on each patient's clinical condi- 
tion. In this review, we aimed to summarize the currently available treatment options and present a therapeutic strategy for NSCLC patients with brain metastases, with particular emphasis on bevacizumab.

\section{Whole-brain radiotherapy}

Whole-brain radiotherapy (WBRT) is the classical treatment approach for brain metastases and the schedule of 10 fractions of 3-Gy over 2 weeks (total dose of $30 \mathrm{~Gy}$ ) is most commonly used. Thus far, eight randomized controlled trials comparing the standard dose schedule (30 Gy divided into 10 fractions) with altered dose schedules have been conducted on patients with brain metastases from various primary cancers, including NSCLC, with no reported significant differences in overall survival (OS) and symptom control rate between the two groups (8).

Concerns have been raised that neurocognitive function may deteriorate following WBRT. According to a previous study, $11 \%$ of patients who received WBRT and survived for 1 year developed severe radiation-induced dementia (9). Another study reported that brain atrophy developed in $\leq 30 \%$ of patients who received $\leq 50$ Gy of WBRT. However, radiographic brain atrophy was not necessarily accompanied by a Mini Mental State Examination (MMSE) score decrease and approximately half of the cases exhibiting a decrease in MMSE scores could be attributed to a decrease in performance status (PS) caused by systemic disease progression (10). Evidence suggests that the deterioration of neurocognitive function is significantly associated with brain tumor growth and the view that the benefits greatly outweigh the disadvantages of WBRT is currently predominant (11-13).

\section{Surgery}

Surgery has occasionally been selected for patients with a single brain metastasis and its role has been established based on randomized controlled trials (Table I) (14-16). In the first study conducted by Patchell et al (14), 48 patients with a single brain metastasis, of whom 37 had NSCLC, were randomized to either surgery followed by WBRT or WBRT alone groups. The frequency of brain metastasis recurrence was significantly reduced in the surgery plus WBRT compared to the WBRT alone group (52 vs. $20 \%$, respectively, $\mathrm{P}<0.02$ ) and the $\mathrm{OS}$ was significantly better in the surgery plus WBRT compared to the WBRT alone group (MST, 40 vs. 15 weeks, respectively; $\mathrm{P}<0.01)$ (14). In the second study, the OS was significantly better in the surgery plus WBRT compared to the WBRT alone group (MST, 43 vs. 26 weeks, respectively; $\mathrm{P}=0.04$ ). However, the survival advantage was prominent in patients with stable extracranial disease (MST, 12 vs. 7 months), whereas in patients with progressive extracranial disease the MST was 5 months in both groups (15). Furthermore, a third study reported no significant differences in survival time: the MST was 6.3 months in the WBRT alone group and 5.6 months in the surgery plus WBRT group $(\mathrm{P}=0.24)(16)$.

Regarding the discrepant results among these studies, it should be noted that $73 \%$ of the patients included in the third study had either extracranial metastases or uncontrollable primary disease, which is higher compared to the other two studies (17). Collectively, surgery followed by WBRT is recommended for NSCLC patients with a single brain metastasis when extracranial disease is controlled.

By contrast, there has been no prospective study of surgery in patients with multiple brain metastases. According to a retrospective study, patients with multiple brain metastases achieved survival times similar to those of patients with a single brain metastasis who underwent surgery, provided all the brain metastases were surgically resected. However, patients with multiple brain metastases who did not have all the brain metastases completely resected exhibited significantly shorter survival times (18). The role of surgery for multiple brain metastases remains uncertain and surgery is not generally recommended for patients with more than one brain metastasis outside a clinical trial.

\section{Radiosurgery}

Stereotactic radiosurgery (SRS) has recently emerged as an alternative option for the treatment of brain metastases. SRS has an advantage over surgical resection: it is less invasive and allows more than one lesion to be treated, including those in areas not surgically accessible.

The largest randomized study comparing WBRT plus SRS and WBRT alone was conducted by the Radiation Therapy Oncology Group (RTOG), in which 333 patients with 1-3 brain metastases, of whom $63 \%$ had lung cancer, were randomly assigned to WBRT plus SRS and WBRT alone groups. No significant survival improvement was observed in the entire population in that study: the MST was 5.7 months in the WBRT alone group and 6.5 months in the WBRT plus $\mathrm{SRS}$ group $(\mathrm{P}=0.1356)$. However, a survival advantage in the WBRT plus SRS over the WBRT alone group was demonstrated in patients with a single brain metastasis (MST, 6.5 vs. 4.9 months, respectively; $\mathrm{P}=0.0393$ ). In addition, patients in the WBRT plus SRS group were more likely to have stable or improved PS at 6-month follow-up compared to patients in the WBRT alone group (43 vs. 27\%, $\mathrm{P}=0.03$ ) (19).

In another study, SRS plus WBRT was compared to SRS alone in patients with 1-4 brain metastases, of whom $67 \%$ had lung cancer, and 132 patients were randomized to each group. The OS was almost identical between the two groups; the MST was 7.5 months in the SRS alone group and 8.0 months in the combination group $(\mathrm{P}=0.42)$. However, the brain tumor recurrence rate at 1 year was significantly lower in the combination group ( 46.8 vs. $76.4 \%, \mathrm{P}<0.001)$. The 1 -year actual rate of developing new brain metastases was also significantly reduced in the combination group ( 41.5 vs. $63.7 \%, \mathrm{P}=0.003$ ). Consequently, salvage brain treatment was less frequently required in the combination group (20).

Therefore, the combination of WBRT and SRS is recommended for NSCLC patients with a single brain metastasis and may be a viable option for patients with $\leq 4$ brain metastases. There has been no direct comparison between surgery and SRS in patients with a single brain metastasis.

\section{Chemotherapy}

The brain has been traditionally considered to be a 'sanctuary' for metastases, under the hypothesis that the blood-brain 
Table I. Randomized studies of whole-brain radiotherapy (WBRT) with or without surgery in solitary brain metastasis.

\begin{tabular}{|c|c|c|c|c|}
\hline Study & Treatment arm & Patient no. & Median survival (weeks) & Refs. \\
\hline \multirow[t]{2}{*}{ Patchell et al } & WBRT alone & 23 & 15 & \multirow[t]{2}{*}{ (14) } \\
\hline & WBRT + surgery & 25 & 40 & \\
\hline \multirow[t]{2}{*}{ Vecht et al } & WBRT alone & 31 & 26 & \multirow[t]{2}{*}{$(15)$} \\
\hline & WBRT + surgery & 32 & 43 & \\
\hline \multirow[t]{2}{*}{ Mintz et al } & WBRT alone & 43 & 6.3 & \multirow[t]{2}{*}{ (16) } \\
\hline & WBRT + surgery & 41 & 5.6 & \\
\hline
\end{tabular}

barrier (BBB), a mechanism found across species that protects the brain from exposure to toxins, limits the delivery of chemotherapeutic agents to the brain (21). Therefore, WBRT has been the standard treatment for NSCLC patients with multiple brain metastases. However, certain studies suggested that patients who have developed brain metastases may have an inherently compromised BBB $(22,23)$.

Robinet et al (24) conducted a randomized phase III study to determine whether the timing of WBRT with respect to chemotherapy affects survival in patients with NSCLC and concurrent brain metastases. Of the patients included in the study, 64\% had multiple brain metastases and $36 \%$ had an inoperable solitary brain metastasis. All the patients received chemotherapy with cisplatin and vinorelbine for a maximum of 6 cycles. A total of 171 eligible patients were randomized to receive chemotherapy alone for at least the first 2 cycles or chemotherapy and early concurrent WBRT (30 Gy divided in 10 fractions). Patients in the chemotherapy alone group received the same WBRT i) at any time in the case of proven clinical progression of brain lesions; ii) after 2 or 4 cycles of chemotherapy in the case of stable brain lesions; and iii) after 6 cycles of chemotherapy, as for other patients. The intracranial response rate was $27 \%$ in the chemotherapy alone group and $33 \%$ in the chemotherapy with concurrent WBRT group $(\mathrm{P}=0.12)$. Survival time was not significantly different between the two groups: the MST was 24 weeks in the chemotherapy alone group and 21 weeks in the chemotherapy with concurrent WBRT group $(\mathrm{P}=0.83)$. Those results suggested that the timing of WBRT does not affect the outcome of NSCLC patients with brain metastases treated with chemotherapy (24).

More recently, Lee et al (25) conducted a similar study in which 48 NSCLC patients with clinically silent and inoperable brain metastases were randomized to upfront chemotherapy followed by WBRT or WBRT followed by chemotherapy groups. Sixty-four percent of the patients had $\geq 3$ brain metastases and the remaining patients had $<3$ brain metastases. The intracranial response rate and the disease control rate were 28.0 and $72.0 \%$ for the chemotherapy first and 39.1 and $56.5 \%$ for the WBRT first arm, respectively. There was no significant difference in survival time: the MST was 9.1 months for the chemotherapy first and 9.9 months for the WBRT first arm $(\mathrm{P}=0.61)$. It is noteworthy that 4 patients $(17.4 \%)$ did not receive further chemotherapy due to early death or poor PS following WBRT in the WBRT first arm (25).
The results of recent retrospective studies have also suggested that upfront chemotherapy is as effective as upfront WBRT in patients with asymptomatic brain metastases (26,27). According to the current European Society of Medical Oncology clinical practice guidelines for metastatic NSCLC, systemic therapy is a viable option for patients with no or relatively minor symptoms from brain metastases with early radiotherapy intervention in the case of the development or progression of symptoms while on treatment (II, B) (28). Overall, upfront chemotherapy may be a reasonable option for NSCLC patients with asymptomatic brain metastases.

\section{Bevacizumab}

Although an intracranial response comparable to the extracranial response has been reported with a pemetrexed-containing regimens, the survival time of patients with brain metastases is still poor compared to stage IV patients without brain metastases $(29,30)$. More effective treatment is required to improve the prognosis of these patients.

Clinical efficacy of bevacizumab in NSCLC. Bevacizumab (Avastin; F. Hoffmann-La Roche, Ltd., Basel, Switzerland) is a humanized monoclonal antibody that inhibits tumor angiogenesis by neutralizing the vascular endothelial growth factor. The first phase III study of bevacizumab in combination with paclitaxel and carboplatin vs. paclitaxel and carboplatin in patients with non-squamous NSCLC (E4599) was conducted in the USA. The progression-free survival (PFS) (6.2 vs. 4.5 months, $\mathrm{P}<0.001)$ and $\mathrm{OS}$ (12.3 vs. 10.3 months, $\mathrm{P}=0.003$ ) were significantly better in the bevacizumab arm (31). In the second phase III study (AVAiL), conducted in the EU, bevacizumab (7.5 and $15 \mathrm{mg} /$ $\mathrm{kg}$ ) was investigated in combination with gemcitabine and cisplatin. PFS, the primary endpoint, was found to be significantly better in the bevacizumab arm (32). According to a recent meta-analysis, bevacizumab in combination with platinum-based chemotherapy significantly prolonged OS and PFS (33). Major clinical guidelines currently recommend the use of bevacizumab in NSCLC patients without contraindications, such as squamous cell histology and history of hemoptysis $(28,34)$.

Safety data of bevacizumab in patients with brain metastases. Despite the promising preclinical data (35-39), patients with brain metastases have been excluded from clinical trials of 

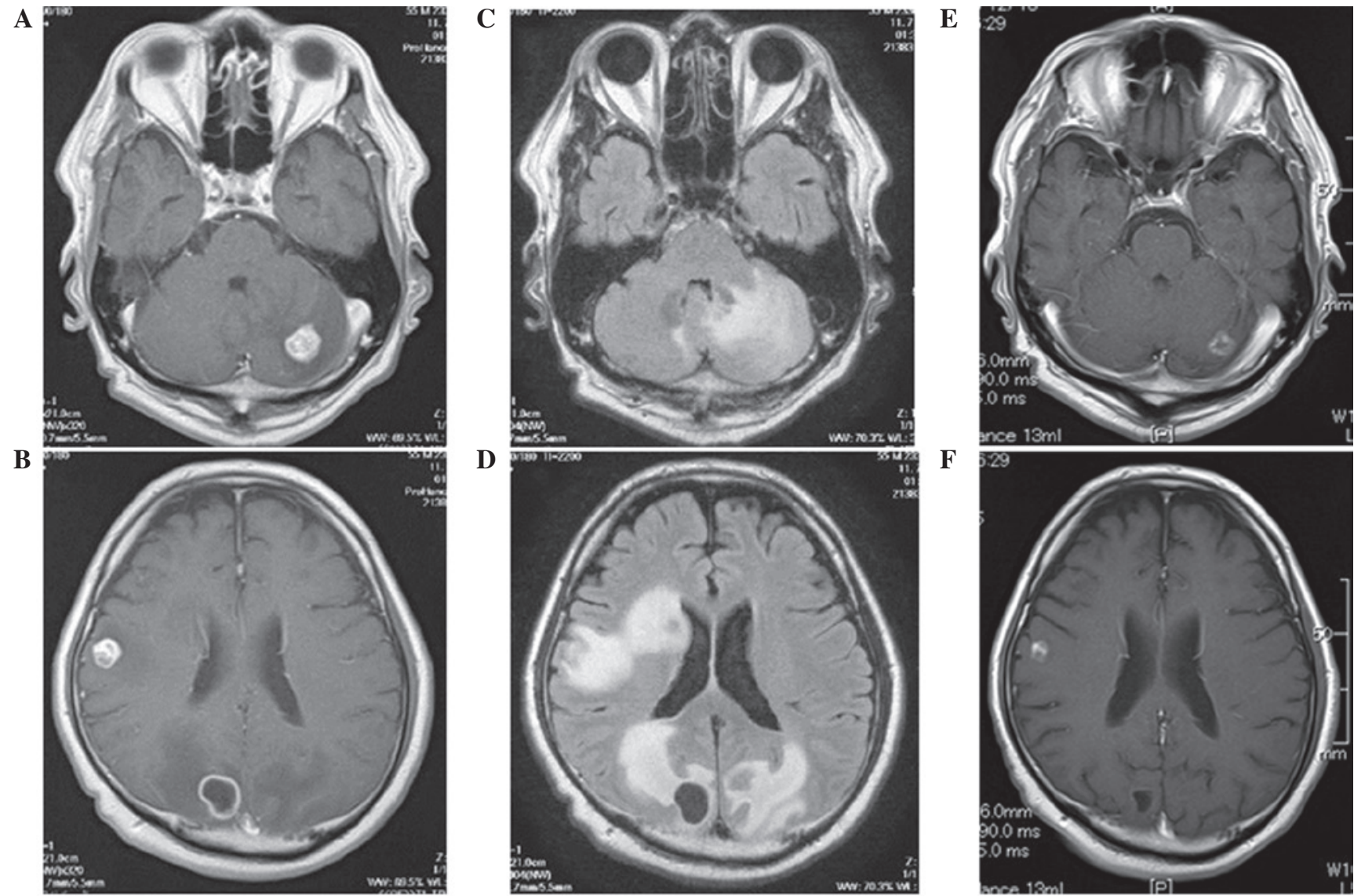

Figure 1. (A and B) Gadolinium-enhanced T1-weighted and (C and D) T2-weighted magnetic resonance imaging (MRI) of the brain prior to chemotherapy with bevacizumab, paclitaxel and carboplatin. (E and F) Gadolinium-enhanced T1-weighted MRI of the brain after 2 cycles of chemotherapy with bevacizumab, paclitaxel and carboplatin.

bevacizumab, following a single serious bleeding event in a phase I trial in one patient with hepatocellular carcinoma and occult brain metastasis (35).

In an attempt to elucidate whether brain metastases are a significant risk factor for intracranial hemorrhage (ICH), Srivastava et al (36) conducted a retrospective analysis of patients with advanced NSCLC using data from the MD Anderson Cancer Center Tumor Registry. This study included 776 patients with and 1,367 patients without brain metastases and the rates of spontaneous ICH were compared. The actual number of patients that developed ICH and the incidence rate (per 1,000 individuals/year) was 9 and 15.5 in patients with brain metastases and 4 and 3.2 in patients without brain metastases, respectively (crude incidence rate ratio, 4.79; $\mathrm{P}=0.0076$ ). However, the actual number of patients developing symptomatic ICH and the incidence rate (per 1,000 individuals/year) were 4 and 6.9 in patients with brain metastases and 4 and 3.2 in patients without brain metastases, respectively (crude incidence rate ratio, 2.13; $\mathrm{P}=0.31$ ). These results demonstrated that the rate of spontaneous ICH appeared to be higher among patients with brain metastases compared to those without, although the rate was very low in both groups and the rates of symptomatic ICH were not significantly different between the two groups (36). A later study by Khasraw et al (37) conducted a retrospective analysis to investigate the association between treatment with bevacizumab and ICH in various types of tumors. It was concluded that bevacizumab does not increase the incidence of ICH in cancer patients, despite the presence of brain metastases. In the NSCLC population, the incidence rate of ICH was $1.00 \%(29 / 2,914)$ in patients treated without bevacizumab and $1.24 \%(3 / 242)$ in patients treated with bevacizumab. Moreover, the incidence rates of ICH were $3.6 \%(28 / 789)$ in patients with brain metastases treated without bevacizumab and 3.9\% (3/77) in patients with brain metastases treated with bevacizumab (37). Retrospective exploratory analyses of randomized controlled trials reported similar results, indicating that bevacizumab is not a significant risk factor for ICH, even in patients with brain metastases $(38,39)$.

In the prospective setting, the safety of bevacizumab for brain metastases was first investigated in patients with treated brain metastases (PASSPORT study) (40). Of the 115 enrolled NSCLC patients, 67 (58.7\%) received WBRT alone, 25 (21.7\%) received WBRT with SRS or surgery, 22 (19.1\%) received SRS alone and $1(0.9 \%)$ received surgery alone. The chemotherapy consisted of carboplatin and paclitaxel (33\%), carboplatin and other agents (28\%), pemetrexed (19\%), erlotinib (10\%) and others (10\%). Among the 106 safety-evaluable patients, there was no reported grade 1-5 ICH (95\% CI: 0.0-3.3\%) (40). Thus, bevacizumab was safely administered to patients with treated brain metastases. In the subsequent study, NSCLC patients with asymptomatic untreated brain metastases were treated with bevacizumab (BRAIN study) (41). In the first-line $\operatorname{arm}(\mathrm{n}=67)$, the combination of bevacizumab, paclitaxel and carboplatin was administered, whereas bevacizumab and erlo- 


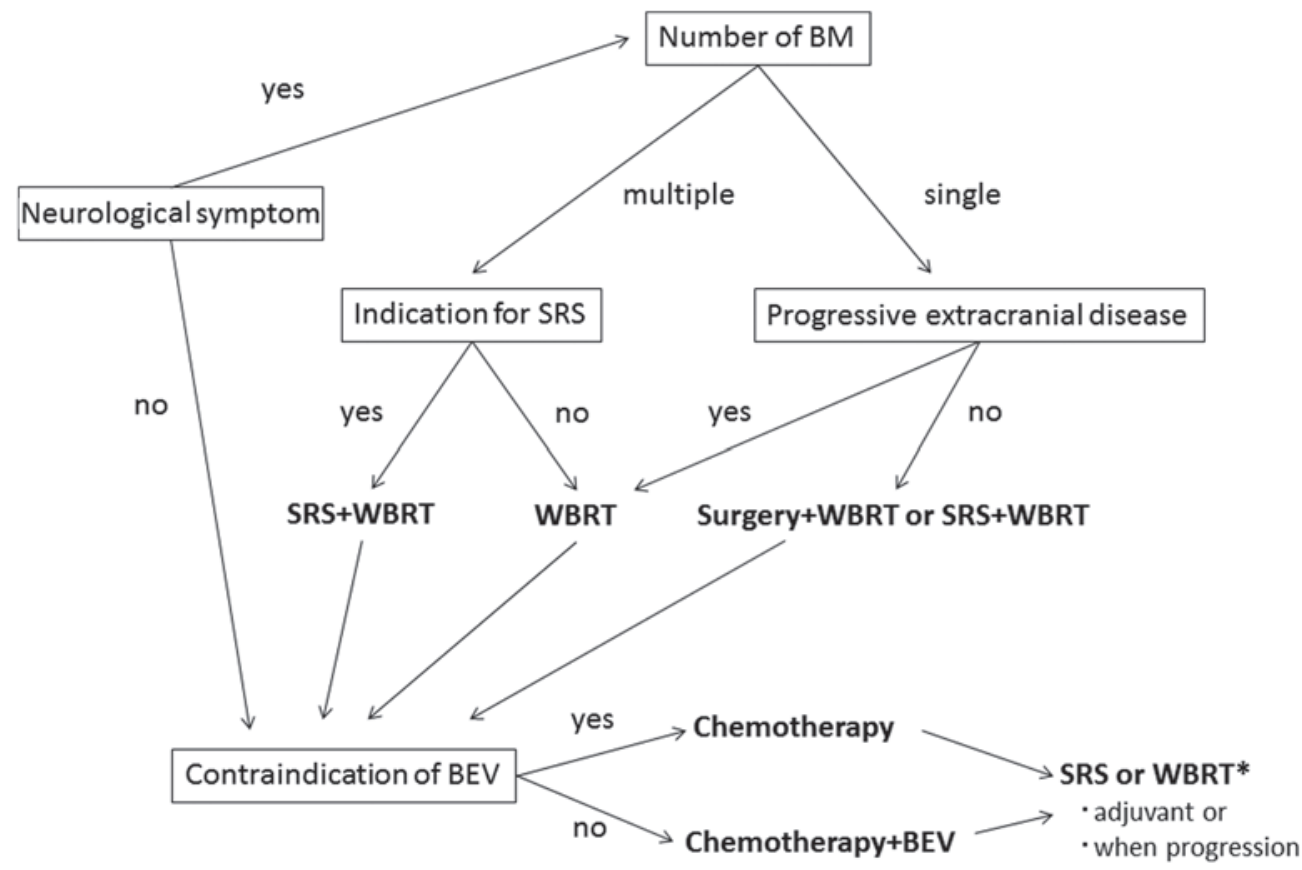

Figure 2. An example of the algorithm for non-small-cell lung cancer patients with brain metastases. "Patients who have previously received WBRT are not usually readministered WBRT. BM, brain metastases; SRS, stereotactic radiosurgery; WBRT, whole-brain radiotherapy; BEV, bevacizumab.

tinib were administered in the second-line arm $(n=24)$. The median PFS was 6.7 months in the first-line and 6.3 months in the second-line arm. The median OS was 15.1 months in the first-line and 13.6 months in the second-line arm. The response rates for intracranial and extracranial metastases were almost identical in the two arms. One grade $1 \mathrm{ICH}$ event as reported in the first-line arm and none were reported in the second-line arm. In this study, bevacizumab exhibited an acceptable safety profile in patients with asymptomatic untreated brain metastases and the survival data were comparable to those of patients without brain metastases treated with bevacizumab (41). Due to the fact that patients with brain metastases generally exhibit lower survival times compared to patients without brain metastases, these data encouraged clinicians to use bevacizumab in this patient population.

There has been no prospective study of bevacizumab in patients with active or symptomatic brain metastases. However, certain case series recently reported that bevacizumab was safe and effective for progressive brain metastases $(42,43)$. A case of progressive brain metastases treated at our hospital is shown in Fig. 1. The patient's brain tumors did not respond to WBRT and he developed seizures. The patient subsequently received a combination chemotherapy with bevacizumab, paclitaxel and carboplatin as second-line chemotherapy and the brain metastases were significantly reduced in size, without ICH. These results suggest that bevacizumab may merit further investigation in patients with active or symptomatic brain metastases as well.

\section{Treatment algorithm for NSCLC patients with brain metastases}

An example of the algorithm for the treatment of NSCLC patients with brain metastases is shown in Fig. 2. Patients with symptomatic brain metastases should be treated with local therapy prior to administration of chemotherapy and the treatment modality should be selected according to the number of brain metastases and the extracranial disease status. Surgery followed by WBRT or SRS+WBRT is recommended for patients with a single brain metastasis. Although there are no established criteria, SRS+WBRT may be considered for patients with $\leq 4$ brain metastases $(\leq 4 \mathrm{~cm})$ and controlled extracranial disease (44). Patients with asymptomatic brain metastases and those who have received prior local therapy should be evaluated for indications for bevacizumab and the administration of bevacizumab is recommended for patients without specific contraindications for this drug.

\section{Conclusions}

In this review we summarized the currently available treatment options for NSCLC patients with brain metastases and highlighted the safety and efficacy of bevacizumab. Considering their dismal prognosis, bevacizumab is recommended particularly for patients with brain metastases. Molecularly-targeted agents were not included in the review to simplify the discussion. Should molecularly-targeted agents be included, the treatment algorithm becomes more complicated. For example, it has not yet been established whether WBRT or tyrosine kinase inhibitor of epidermal growth factor receptor (EGFR) is preferred as initial treatment for patients with activating mutation of EGFR, or which treatment option is preferred for neurologically symptomatic patients. There remain several unanswered questions regarding the treatment of NSCLC patients with brain metastases. A more sophisticated treatment strategy, including molecularly-targeted agents, should be identified to cater for personalized medicine. 


\section{References}

1. SocinskiMA, Crowell R, Hensing TE, et al: Treatment of non-small cell lung cancer, stage IV: ACCP evidence-based clinical practice guidelines (2nd edition). Chest 132: S277-S289, 2007

2. Nayak L, Lee EQ and Wen PY: Epidemiology of brain metastases. Curr Oncol Rep 14: 48-54, 2012.

3. Shi AA, Digumarthy SR, Temel JS, et al: Does initial staging or tumor histology better identify asymptomatic brain metastases in patients with non-small cell lung cancer? J Thorac Oncol 1: 205-210, 2006

4. Gaspar L, Scott C, Rotman M, Asbell S, Phillips T, Wasserman T, McKenna WG and Byhardt R: Recursive partitioning analysis (RPA) of prognostic factors in three Radiation Therapy Oncology Group (RTOG) brain metastases trials. Int J Radiat Oncol Biol Phys 37: 745-751, 1997.

5. Penel N, Brichet A, Prevost B, et al: Prognostic factors of synchronous brain metastases from lung cancer. Lung Cancer 33: 143-154, 2001.

6. Ampil F, Caldito G, Milligan S, et al: The elderly with synchronous non-small cell lung cancer and solitary brain metastasis: does palliative thoracic radiotherapy have a useful role? Lung Cancer 57: 60-65, 2007.

7. Sanchez de Cos J, Sojo Gonzalez MA, Montero MV, et al: Non-small cell lung cancer and silent brain metastasis. Surviva and prognostic factors. Lung Cancer 63: 140-145, 2009.

8. Tsao MN, Lloyd N, Wong RK, et al: Whole brain radiotherapy for the treatment of newly diagnosed multiple brain metastases. Cochrane Database Syst Rev 4: CD003869, 2012.

9. DeAngelis LM, Mandell LR, Thaler HT, et al: The role of postoperative radiotherapy after resection of single brain metastases. Neurosurgery 24: 798-805, 1989.

10. Shibamoto Y, Baba F, Oda K, et al: Incidence of brain atrophy and decline in mini-mental state examination score after whole-brain radiotherapy in patients with brain metastases: a prospective study. Int J Radiat Oncol Biol Phys 72: 1168-1173, 2008.

11. Meyers CA, Smith JA, Bezjak A, et al: Neurocognitive function and progression in patients with brain metastases treated with whole-brain radiation and motexafin gadolinium: results of a randomized phase III trial. J Clin Oncol 22: 157-165, 2004.

12. Li J, Bentzen SM, Renschler M and Mehta MP: Regression after whole-brain radiation therapy for brain metastases correlates with survival and improved neurocognitive function. J Clin Oncol 25: 1260-1266, 2007.

13. Tallet AV, Azria D, Barlesi F, et al: Neurocognitive function impairment after whole brain radiotherapy for brain metastases: actual assessment. Radiat Oncol 7: 77, 2012.

14. Patchell RA, Tibbs PA, Walsh JW, et al: A randomized trial of surgery in the treatment of single metastases to the brain. $\mathrm{N}$ Engl J Med 322: 494-500, 1990.

15. Vecht CJ, Haaxma-Reiche H, Noordijk EM, et al: Treatment of single brain metastasis: radiotherapy alone or combined with neurosurgery? Ann Neurol 33: 583-590, 1993.

16. Mintz AH, Kestle J, Rathbone MP, et al: A randomized tria to assess the efficacy of surgery in addition to radiotherapy in patients with a single cerebral metastasis. Cancer 78: 1470-1476, 1996.

17. Wronski $\mathrm{M}$ and Lederman G: A randomized trial to assess the efficacy of surgery in addition to radiotherapy in patients with a single cerebral metastasis. Cancer 80: 1002-1004, 1997.

18. Bindal RK, Sawaya R, Leavens ME and Lee JJ: Surgical treatment of multiple brain metastases. J Neurosurg 79: 210-216, 1993.

19. Andrews DW, Scott CB, Sperduto PW, et al: Whole brain radiation therapy with or without stereotactic radiosurgery boost for patients with one to three brain metastases: phase III results of the RTOG 9508 randomised trial. Lancet 363: 1665-1672, 2004.

20. Aoyama H, Shirato H, Tago M, et al: Stereotactic radiosurgery plus whole-brain radiation therapy vs. stereotactic radiosurgery alone for treatment of brain metastases: a randomized controlled trial. JAMA 295: 2483-2491, 2006.

21. Walbert T and Gilbert MR: The role of chemotherapy in the treatment of patients with brain metastases from solid tumors. Int J Clin Oncol 14: 299-306, 2009.

22. Deeken JF and Loscher W: The blood-brain barrier and cancer: transporters, treatment, and Trojan horses. Clin Cancer Res 13: $1663-1674,2007$

23. Mehta MP, Paleologos NA, Mikkelsen T, et al: The role of chemotherapy in the management of newly diagnosed brain metastases: a systematic review and evidence-based clinical practice guideline. J Neurooncol 96: 71-83, 2010.
24. Robinet G, Thomas P, Breton JL, et al: Results of a phase III study of early versus delayed whole brain radiotherapy with concurrent cisplatin and vinorelbine combination in inoperable brain metastasis of non-small-cell lung cancer: Groupe Français de PneumoCancérologie (GFPC) Protocol 95-1. Ann Oncol 12: 59-67, 2001

25. Lee DH, Han JY, Kim HT, et al: Primary chemotherapy for newly diagnosed nonsmall cell lung cancer patients with synchronous brain metastases compared with whole-brain radiotherapy administered first: result of a randomized pilot study. Cancer 113 143-149, 2008.

26. Moscetti L, Nelli F, Felici A, et al: Up-front chemotherapy and radiation treatment in newly diagnosed nonsmall cell lung cancer with brain metastases: survey by Outcome Research Network for Evaluation of Treatment Results in Oncology. Cancer 109: 274-281, 2007.

27. Kim KH, Lee J, Lee JI, et al: Can upfront systemic chemotherapy replace stereotactic radiosurgery or whole brain radiotherapy in the treatment of non-small cell lung cancer patients with asymptomatic brain metastases? Lung Cancer 68: 258-263, 2010.

28. Peters S, Adjei AA, Gridelli C, et al: Metastatic non-small-cell lung cancer (NSCLC): ESMO Clinical Practice Guidelines for diagnosis, treatment and follow-up. Ann Oncol 23 (Suppl 7): vii56-vii64, 2012.

29. Barlesi F, Gervais R, Lena H, et al: Pemetrexed and cisplatin as first-line chemotherapy for advanced non-small-cell lung cancer (NSCLC) with asymptomatic inoperable brain metastases: a multicenter phase II trial (GFPC 07-01). Ann Oncol 22: 2466-2470, 2011.

30. Bailon O, Chouahnia K, Augier A, et al: Upfront association of carboplatin plus pemetrexed in patients with brain metastases of lung adenocarcinoma. Neuro Oncol 14: 491-495, 2012.

31. Sandler A, Gray R, Perry MC, et al: Paclitaxel-carboplatin alone or with bevacizumab for non-small-cell lung cancer. N Engl J Med 355: 2542-2550, 2006.

32. Reck M, von Pawel J, Zatloukal P, et al: Overall survival with cisplatin-gemcitabine and bevacizumab or placebo as first-line therapy for nonsquamous non-small-cell lung cancer: results from a randomised phase III trial (AVAiL). Ann Oncol 21: 1804-1809, 2010.

33. Soria JC, Mauguen A, Reck M, et al: Systematic review and meta-analysis of randomised, phase II/III trials adding bevacizumab to platinum-based chemotherapy as first-line treatment in patients with advanced non-small-cell lung cancer. Ann Oncol 24: 20-30, 2013.

34. Azzoli CG, Baker S Jr, Temin S; American Society of Clinical Oncology: American Society of Clinical Oncology Clinical Practice Guideline update on chemotherapy for stage IV non-small-cell lung cancer. J Clin Oncol 27: 6251-6266, 2009.

35. Gordon MS, Margolin K, Talpaz M, et al: Phase I safety and pharmacokinetic study of recombinant human anti-vascular endothelial growth factor in patients with advanced cancer. J Clin Oncol 19: 843-850, 2001.

36. Srivastava G, Rana V, Wallace S, et al: Risk of intracranial hemorrhage and cerebrovascular accidents in non-small cell lung cancer brain metastasis patients. J Thorac Oncol 4: 333-337, 2009.

37. Khasraw M, Holodny A, Goldlust SA and DeAngelis LM: Intracranial hemorrhage in patients with cancer treated with bevacizumab: the Memorial Sloan-Kettering experience. Ann Oncol 23: 458-463, 2012.

38. Carden CP, Larkin JM and Rosenthal MA: What is the risk of intracranial bleeding during anti-VEGF therapy? Neuro Oncol 10: 624-630, 2008

39. Besse B, Lasserre SF, Compton P, et al: Bevacizumab safety in patients with central nervous system metastases. Clin Cancer Res 16: 269-278, 2009.

40. Socinski MA, Langer CJ, Huang JE, et al: Safety of bevacizumab in patients with non-small-cell lung cancer and brain metastases. $\mathrm{J}$ Clin Oncol 27: 5255-5261, 2009.

41. Besse B, Le Moulec S, Senellart H, et al: Phase II study of bevacizumab in combination with first-line chemotherapy or second-line erlotinib in non-squamous NSCLC patients with asymptomatic untreated brain metastases (ML21823). Ann Oncol 23: ix426, 2012.

42. De Braganca KC, Janjigian YY, Azzoli CG, et al: Efficacy and safety of bevacizumab in active brain metastases from non-small cell lung cancer. J Neurooncol 100: 443-447, 2010.

43. Yamamoto D, Iwase S, Tsubota Y, et al: Bevacizumab in the treatment of five patients with breast cancer and brain metastases: Japan Breast Cancer Research Network-07 trial. Onco Targets Ther 5: 185-189, 2012

44. Tsao MN, Lloyd NS and Wong RK; Supportive Care Guidelines Group of Cancer Care Ontario's Program in Evidence-based Care: Clinical practice guideline on the optimal radiotherapeutic management of brain metastases. BMC Cancer 5: 34, 2005. 